\title{
Clinical Significance of Granulation Pattern in Corticotroph Pituitary Adenomas
}

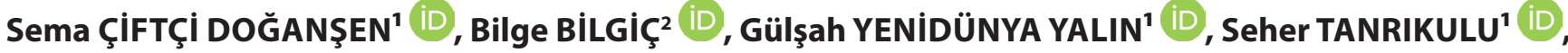 \\ Sema YARMAN ${ }^{1}$ iD
}

Department of 'Internal Medicine, Division of Endocrinology and Metabolism, ${ }^{2}$ Pathology, Istanbul University, Istanbul Faculty of Medicine, ISTANBUL, TURKEY

\section{ABSTRACT}

Objective: The granulation pattern of pituitary tumors may be important to predict tumor behavior, treatment outcomes, and recurrences. Therefore, we compared densely granulated corticotroph tumor (DGCT) and sparsely granulated corticotroph tumor (SGCT) in terms of clinicopathologic findings.

Material and Method: A total of 41 patients (31 females/10 males) were assessed in the study. The granulation patterns were detected by Periodic Acid-Schiff (PAS) and adrenocorticotropic hormone (ACTH) immunoreactivity. Diffuse and strong staining by PAS and ACTH were identified as DGCT, whereas faint staining by PAS and focal, weak staining by ACTH were identified as SGCT. Perinuclear ring-like patterns with low molecular weight keratin were identified as Crooke's cell tumors. DGCTs and SGCTs were compared in terms of clinical findings and treatment outcomes.

Results: The majority of the patients (71\%) had DGCTs ( $\mathrm{n}=29)$; whereas $24 \%$ of the patients had SGCTs ( $\mathrm{n}=10)$ and $5 \%$ of the patients had Crooke's cell tumors $(n=2)$. DGCTs and SGCTs were similar in terms of age, sex, estimated duration of disease, baseline cortisol and ACTH levels. SGCTs were larger than DGCTs ( $\mathrm{p}=0.034$ ). The remissions with surgery rates were similar between the groups; however, hypothalamic-pituitaryadrenal axis recovery time was longer in SGCTs $(\mathrm{p}=0.033)$. Persistent disease after surgery was slightly higher in DGCTs and recurrences were frequent in SGCTs; however, the differences were not statistically significant.

Conclusion: DGCTs are often small microadenomas, while SGCTs are larger. Recurrences were slightly more common in SGCTs; however, further studies including larger series are needed to be able to obtain more significant results.

Key Words: Cushing's disease, Corticotroph tumor, Densely granulated, Sparsely granulated, Recurrence

\section{INTRODUCTION}

Cushing's disease $(\mathrm{CD})$ is an aggressive endocrine disorder caused by a pituitary tumor secreting adrenocorticotrophic hormone (ACTH) and may lead to significant morbidity and mortality (1). Despite significant advances in the physiopathology and treatment options, there are still many challenges in the management of $\mathrm{CD}$. The primary treatment is surgery; however, persistent disease and recurrences after the surgery are common (2).

Histological findings in the specimens obtained from surgery and the granulation pattern of the tumors may help predict disease recurrence (3). Although corticotroph tumors have not been classified in accordance with granulation patterns in the World Health Organization (WHO) 2004 criteria, dense and sparse granulation patterns of prolactinomas and somatotroph tumors have widely

(Turk Patoloji Derg 2019, 35:9-14)

Received : 21.03.2018 Accepted : 16.06.2018 been identified (4). For this reason, granulation patterns of corticotroph tumors have been included in the latest World Health Organization classification in 2017 (5). In line with this, corticotroph tumors are grouped as densely or sparsely granulated according to Periodic Acid-Schiff (PAS) and ACTH immunoreactivity characteristics (3, 6-9). It has been reported that densely granulated corticotroph tumors (DGCT) were detected in smaller adenomas with a calmer clinical course, whereas sparsely granulated corticotroph tumors (SGCT) were detected in larger, more aggressive and invasive tumors (3, 6-8). Furthermore, Crooke's cell tumors constitute another subtype, which is relatively rare and is considered to be more aggressive than others $(9,10)$.

Given the scarcity of the studies correlating clinical and laboratory findings on the tumor granulation pattern in $\mathrm{CD}$, we aimed to investigate the clinicopathological characteristics of DGCTs and SGCTs in this study.

Correspondence: Sema ÇİFTÇí DOĞANŞEN

Istanbul University, Istanbul Faculty of Medicine, Department of Internal Medicine, Division of Endocrinology and Metabolism, İSTANBUL, TURKEY E-mail: sdogansen@gmail.com Phone: +90212 2142000 / 1215 


\section{MATERIAL and METHODS}

Among a total of 320 patients, who were diagnosed with functional pituitary tumor and followed-up at the Istanbul Medical Faculty pituitary out-patient clinic, 54 patients (17\%) were diagnosed with CD. Patients with an accurate histological re-evaluation of tumor subtype analysis were included in this study $(n=41)$. The diagnosis of CD was based on the clinical and radiological findings (pituitary tumor by sellar magnetic resonance imaging-MRI) and laboratory tests. The laboratory diagnosis of $\mathrm{CD}$ was confirmed by failure to suppress plasma cortisol levels $(>1.8 \mu \mathrm{g} / \mathrm{dl})$ after administration of $1 \mathrm{mg}$-overnight and low dose dexamethasone suppression tests (48 hours, 2 $\mathrm{mg}$ /day), high ACTH levels and suppressed cortisol levels with the high dose dexamethasone test $(>50 \%$ suppression from baseline serum cortisol levels) in accordance with the current guideline (1).

The surgical method performed on all patients was transsphenoidal surgery (TSS). The patients with ACTH positive immunostaining adenoma were enrolled in the study, whereas the patients with a pathology result of hyperplasia or normal pituitary tissue or ACTH-negative staining were excluded from the study. All pathological specimens were re-evaluated by an experienced pathologist (BB). Initial evaluation was performed by hematoxylineosin and subsequently silver stain; those with a preserved acinar reticular pattern were excluded as expected with normal pituitary tissue or hyperplasia. The PAS stain was used to identify corticotroph cells. Diffuse and strong staining by PAS and ACTH were identified as DGCT, whereas faint staining by PAS and focal, weak staining by ACTH were identified as SGCT $(3,6-10)$. Typical abundant perinuclear ring-like patterns with low molecular weight keratin (LMWK) were identified as Crooke's cell tumors (3, 6-10). Examples of granulation pattern and MRI from the patient cohort are shown in Figure 1A-F;2A,B.

DGCTs and SGCTs were compared in terms of age, sex, maximum tumor diameter, estimated duration of $\mathrm{CD}$ (calculated according to the information in the patient's history), baseline cortisol, ACTH levels and Ki-67 labeling index. Crooke's cell tumors were excluded from evaluation due to the low number of patients available for these comparisons. The Ki-67 labeling index was evaluated through MIB1 antibody in the tumor tissue samples. The Ki-67 labeling index was assessed by the eye-balling method and when the index was over 1-2\%, approximately 100 nuclei of the tumor cells at $\mathrm{x} 400$ magnification were counted in the evaluation of the Ki-67 score.
Remission rates and persistent hypercortisolism after TSS were compared between the groups. A plasma cortisol level of $<2 \mu \mathrm{g} / \mathrm{dL}$ in the first week after TSS and no recurrence during the follow-up period were determined as remission with TSS and these were compared with the hypothalamic-pituitary-adrenal (HPA) axis recovery time. HPA axis recovery was evaluated by morning cortisol and/ or ACTH stimulation tests. Prednisolone replacement was discontinued when morning plasma cortisol levels were $\geq 10 \mu \mathrm{g} / \mathrm{dL}$ or stimulated cortisol levels were approximately $\geq 18 \mu \mathrm{g} / \mathrm{dL}$ with the ACTH stimulation test (2). A plasma cortisol level $>2 \mu \mathrm{g} / \mathrm{dL}$ on the first week after TSS was determined as persistent hypercortisolism and recurrence was defined in patients who developed clinical and laboratory signs of hypercortisolemia during the follow-up period $(2,11)$. At least one of the options of repeat surgery, medical treatment, radiotherapy (RT) and bilateral adrenalectomy was applied during the follow-up period for persistent disease or recurrence, and the remission rates at the last visit were compared under these treatments.

\section{RESULTS}

The majority of the patients $(71 \%)$ had DGCTs $(n=29)$; whereas $24 \%$ of the patients had SGCTs $(n=10)$ and $5 \%$ of the patients had Crooke's cell tumors $(n=2)$. DGCTs and SGCTs groups were similar in terms of age, sex, baseline cortisol and ACTH levels, whereas SGCTs were larger and the numbers of macroadenomas at borderline were higher than DGCTs ( $\mathrm{p}=0.034, \mathrm{p}=0.05$, respectively). Estimated duration of CD was longer in SGCTs; however, the difference was not statistically significant.

Rates of remission with TSS were similar between the groups whereas HPA axis recovery time was longer in SGCTs ( $\mathrm{p}=0.033)$; however, rates of permanent hypocortisolism without adrenalectomy were similar. Persistent disease after TSS was slightly higher in DGCTs and recurrences were frequent in SGCTs; however, the differences were not statistically significant. The remission rates at the last visit were similar in the groups with similar mean follow-up periods and the Ki-67 labeling indexes of the tumors were also similar. Detailed data about comparison of DGCTs and SGCTs are shown in Table I.

\section{DISCUSSION}

In our series, the majority of corticotroph tumors were DGCT. It is reported in the literature that the majority of corticotroph tumors were densely granulated $(3,6-8,10,12)$. However, in a recent study, Mete et al. (13) reported the frequency of SGCTs and DGCTs in their cohort as $29 \%$ and $47 \%$, respectively and interpreted this result as an 

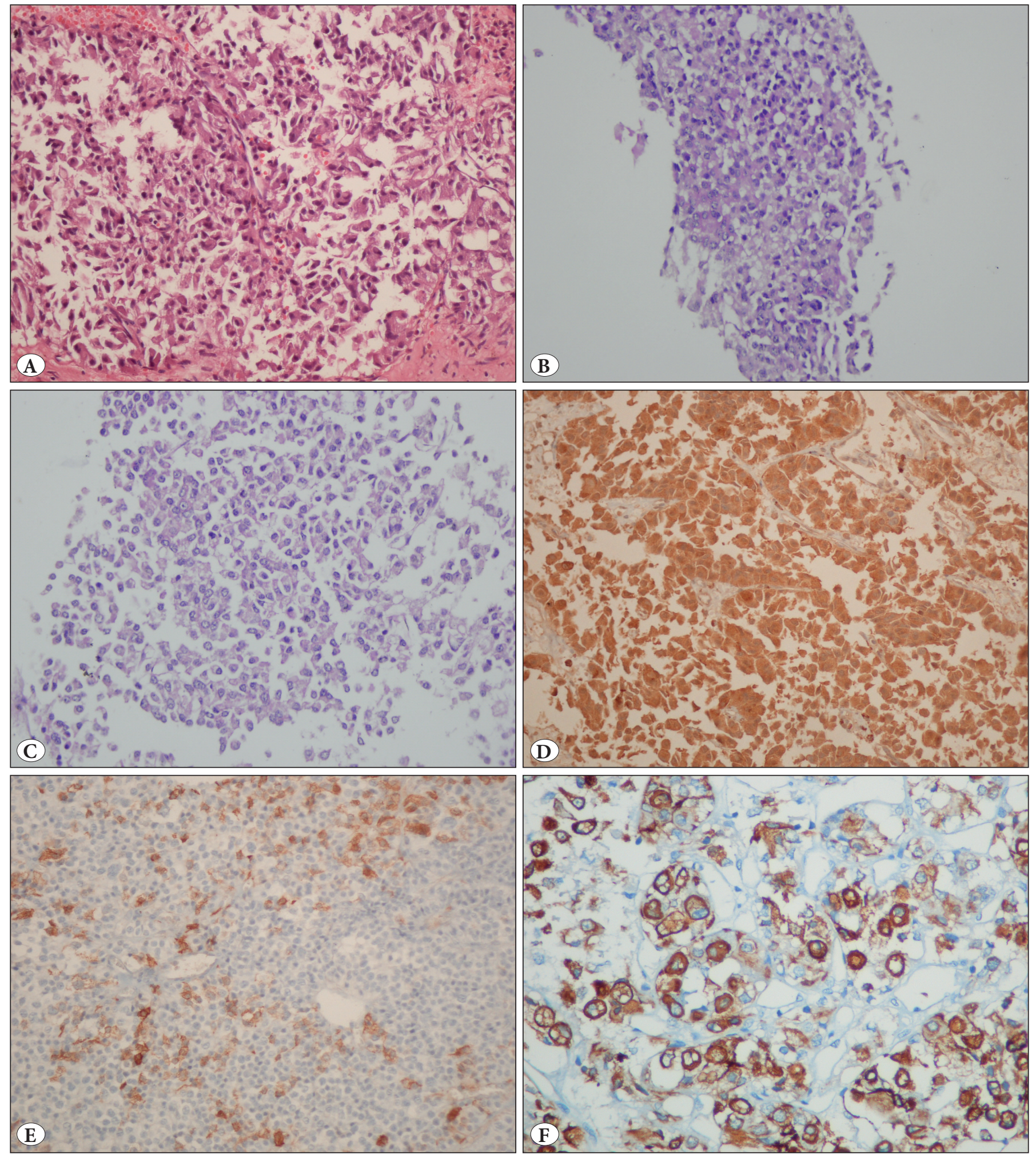

Figure 1: Histological, histochemical and immunohistochemical features of corticotroph tumors. A) Microscopic view of pituitary adenoma (H\&E; x200). B) Densely granulated corticotroph tumor dominated by the tumor cells with diffuse and strong PAS staining (PAS; x100) C) Sparsely granulated corticotroph tumor dominated by the tumor cells with focal and weak PAS staining (PAS; x200). D) Densely granulated corticotroph tumor dominated by the tumor cells with diffuse and strong ACTH staining (IHC; x200). E) Sparsely granulated corticotroph tumor dominated by the tumor cells with focal and weak ACTH staining (IHC; x200). F) Crooke's hyaline changes with cytokeratin staining as ring-like pattern on corticotroph cells (IHC; x400). 
interesting finding. However, they explained these findings with the relatively high frequency of Crooke's cell tumors ( $13 \%$ of corticotroph tumors), the identification of mixed densely and sparsely granulated corticotroph tumors in some patients, and the role of p27 immunohistochemistry in the prediction of corticotroph tumor functionality (13).

In our study, we focused on the association between granulation pattern of corticotroph tumors and the baseline findings of the disease. Accordingly, the groups were similar in terms of sex distribution and, as expected, female dominance existed in both groups. Although age at diagnosis was slightly increased in the SGCTs, the difference was not significant and this result was similar to a recent study (13). Early diagnosis of CD has effectively reduced the mortality rates and prevented the severe comorbidities observed in these patients $(14,15)$. For this reason, we evaluated the duration until diagnosis of CD based on the patient information and findings; and compared the groups to assess the association between granulation pattern and indolent disease period. According to this, while this duration was slightly longer in SGCTs, the difference was not statistically significant, and if patient numbers were increased, more meaningful results could be obtained. We concluded that the CD findings are slower and insidious in SGCTs. A negative correlation between
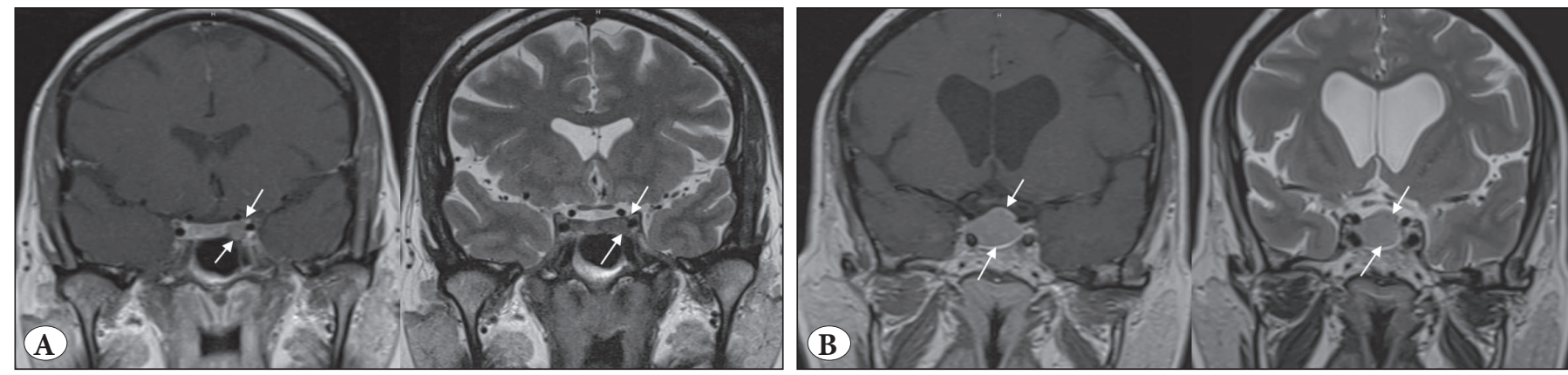

Figure 2: Magnetic resonance imaging features of corticotroph tumors. A) T1 and T2 weighted sequence images of a corticotroph microadenoma; 6x9 mm microadenoma involving the left side of the pituitary gland (white arrow). B) T1 and T2 weighted sequence images of a corticotroph macroadenoma; 22x16 mm macroadenoma involving the pituitary gland (white arrow).

Table I: Distribution of clinical, laboratory data and treatment responses in corticotroph adenomas according to the granulation pattern.

\begin{tabular}{|c|c|c|c|}
\hline $\mathrm{N}=39$ & $\begin{array}{l}\text { Densely granulated } \\
\qquad(\mathbf{n}=29)\end{array}$ & $\begin{array}{l}\text { Sparsely granulated } \\
\qquad(\mathrm{n}=10)\end{array}$ & $\mathbf{p}$ \\
\hline $\begin{array}{l}\text { Age at diagnosis (years) } \\
\text { mean } \pm \mathrm{SD} \text { (range) }\end{array}$ & $34 \pm 13$ & $38 \pm 13$ & NS \\
\hline Sex (Female/Male) & $23 / 6$ & $8 / 2$ & NS \\
\hline Estimated duration of CD (months) & $26 \pm 19$ & $34 \pm 31$ & NS \\
\hline Maximal tumor diameter $(\mathrm{mm})$ mean $\pm \mathrm{SD}$ & $7.9 \pm 6.1$ & $10 \pm 4.7$ & 0.034 \\
\hline Micro- / macroadenomas (n / n) & $19 / 10$ & $3 / 7$ & 0.05 \\
\hline Baseline cortisol levels $(\mu \mathrm{g} / \mathrm{dL})$ mean $\pm \mathrm{SD}$ & $26 \pm 10$ & $27 \pm 11$ & NS \\
\hline Baseline ACTH levels $(\mathrm{pg} / \mathrm{mL})$ mean \pm SD & $78 \pm 51$ & $107 \pm 74$ & NS \\
\hline Remission with TSS (n; \%) & $17(59)$ & $6(60)$ & NS \\
\hline HPA axis recovery time (months) mean $\pm \mathrm{SD}$ & $7.9 \pm 6.3$ & $18 \pm 12$ & 0.033 \\
\hline Permanent hypocortisolism without adrenalectomy $(\mathrm{n} ; \%)$ & $3(10)$ & $2(20)$ & NS \\
\hline Persistent disease $(\mathrm{n} ; \%)$ & $8(28)$ & $1(10)$ & NS \\
\hline Recurrence (n; \%) & $4(14)$ & $3(30)$ & NS \\
\hline Ki-67 labelling index & $1.6 \pm 1.4$ & $2.4 \pm 1.1$ & NS \\
\hline Duration of follow-up (months) mean \pm SD & $96 \pm 53$ & $74 \pm 44$ & NS \\
\hline Remission rate at last visit (n; \%) & $25(86)$ & $8(80)$ & NS \\
\hline
\end{tabular}

$\mathrm{P}<0.05$ statistically significant; Significant $\mathrm{p}$ value is shown in bold.

ACTH: adrenocorticotropic hormone, CD: Cushing's disease, HPA: hypothalamic-pituitary-adrenal , TSS: transsphenoidal surgery, 
the tumor diameter and symptomatology in corticotroph tumors have been well adapted with a view of $\mathrm{CD}$ as "small tumor, big Cushing; big tumor, small Cushing" (8). Similarly, tumor sizes were larger in SGCTs in our patients; and as expected macroadenomas were more frequent in this group. It was reported that SGCTs were larger, invasive tumors and small microadenomas are frequently densely granulated (3, 6-8). Despite larger tumor size in SGCTs, baseline ACTH and cortisol levels were similar between the groups; however, the similarity could be due to the low number of patients in the groups.

Treatment goals in CD are to normalize cortisol secretion, to prevent hypercortisolemia associated morbidities and complications; and to provide a long-term remission without recurrence (16). Since it is not always possible to achieve and maintain these goals, we aimed to assess the effect of the granulation pattern in achieving these treatment goals. Remission with TSS and rates of persistent disease after surgery were similar in these patients who were treated with primary surgery. Normally, maximum tumor diameter in SGCTs was larger; therefore, lower remission or higher persistent disease rates could be expected in SGCTs, and due to the presence of more microadenomas in DGCTs, higher remission rates could be expected in these patients. The reason for the similar frequencies is possibly due to the insufficient detection of small microadenomas by the surgeon as one of the most important predictors of surgical outcomes in $\mathrm{CD}$ is pre-operative visualization of pituitary tumors $(16,17)$. Furthermore, although SGCTs have more macroadenomas, the mean tumor size was about 1 centimeter. It has been explained that remission rates were lower in the tumors over 2 centimeters (18). HPA axis recovery time was longer in SGCTs after the surgery; however, post-operative frequencies of permanent hypocortisolism were similar.

In addition to persistent hypercortisolism, recurrences may occur during follow-up in patients with initial remission after surgery, and a large-scale review has determined the mean recurrence frequency as $13 \%$. (16). As we have already mentioned, it has been stated that recurrences or Nelson's syndrome is more frequent in SGCTs despite the fact that there is no study comparing the rates of recurrence $(3,6-9)$. In our study, recurrence rates were determined as 2 -fold higher in SGCTs within similar follow-up durations (14\% and $30 \%)$, but the difference was not statistically significant. Thus, we considered that these results may be related to the small size of the study groups. We think that the subtype of corticotroph tumors is important in predicting the recurrence, and closer monitoring is therefore required. Presence of Crooke's hyaline changes in adjacent non-tumoral tissue confirms the presence of hypercortisolemia and the presence or absence of Crook's hyaline changes with complete or incomplete pattern properties, which may also be used in predicting remission $(8,19)$. However, as the specimens did not contain adequate adjacent adenohypophysis tissue, the presence or absence of Crooke's cell changes in the non-tumoral corticotrophs could not be evaluated in this study. Further studies with larger pathological specimens may be helpful in the evaluation of the relationship between Crooke's hyaline changes and tumor granulation patterns. The absence of this finding may indicate a more severe defect in the feedback regulation of corticotrophs, which may be related with an increased rate of recurrence $(8,19)$.

It was demonstrated in somatotroph tumors that the granulation pattern of the tumor is crucial in determining the response to somatostatin analog therapy (20). However, such data does not exist for corticotroph tumors in the literature. In our study, there were not enough patients receiving pituitary directed medical treatment such as pasireotide or cabergoline in a long-term period, and such an evaluation could therefore not be performed. We believe that the present study would guide future studies in prediction of a granulation pattern in response to medical therapy, especially as the use of pasireotide will become more common.

It was previously reported that SGCTs are more invasive and aggressive. Some indexes such as Ki-67, p53, an loss of p27 are used for behavioral detection of pituitary tumors $(12,13,21,22)$. We assessed the Ki-67 labeling index in corticotroph tumors with sufficient samples only. Despite slightly higher averages of Ki-67 in SGCTs, the difference was not statistically significant; and similar results were shown in the study reported by Witek et al. (12). However, the eye-balling method we used to evaluate $\mathrm{Ki}-67$ is no longer accepted in the field of neuroendocrine neoplasms including those of pituitary origin and also a minimum of 500 tumor cells should be counted for this examination. This is a deficit of our study, and it may be the reason why the results were not statistically significant.

In conclusion, DGCTs are common in CD. DGCTs have a small microadenoma pattern in comparison to SGCTs, the indolent period is slightly longer, and post-operative HPA axis recovery requires more time. Recurrences are slightly more common in SGCTs; however, further studies with larger series are needed to obtain more significant results. 


\section{ACKNOWLEDGEMENT}

The authors highly appreciate the efforts of Assistant Professor Feyza Nur Tuncer (Istanbul University Aziz Sancar Institute of Experimental Medicine, Department of Genetics) in the language editing of this paper.

\section{CONFLICT of INTEREST}

The authors declare that they have no conflict of interest.

\section{REFERENCES}

1. Nieman LK, Biller BM, Findling JW, Newell-Price J, Savage MO, Stewart PM, Montori VM. The diagnosis of Cushing's syndrome: An Endocrine Society Clinical Practice Guideline. J Clin Endocrinol Metab. 2008;93:1526-40.

2. Nieman LK, Biller BM, Findling JW, Murad MH, Newell-Price J, Savage MO, Tabarin A; Endocrine Society. Treatment of Cushing's Syndrome: An Endocrine Society Clinical Practice Guideline. J Clin Endocrinol Metab. 2015;100:2807-31.

3. Syro LV, Rotondo F, Cusimano MD, Di Ieva A, Horvath E, Restrepo LM, Wong M, Killinger DW, Smyth H, Kovacs K. Current status on histological classification in Cushing's disease. Pituitary. 2015;18:217-24.

4. World Health Organization Classification of Tumours. Pathology and Genetics of Tumours of Endocrine Organs. DeLellis RA, Lloyd RV, Heitz PU, Eng C (eds). Lyon: IARC Press, 2004.

5. World Health Organization Classification of Tumours of Endocrine Organs. Lloyd RV, Osamura RY, Klöppel G, Rosai J (eds). $4^{\text {th }}$ ed. Lyon: IARC Press, 2017

6. Asa SL, Ezzat S. The pathogenesis of pituitary tumors. Annu Rev Pathol 2009;4:97-126.

7. Saeger W, Honegger J, Theodoropoulou M, Knappe UJ, Schöfl C, Petersenn S, Buslei R. Clinical Impact of the Current WHO Classification of Pituitary Adenomas. Endocr Pathol. 2016;27:104-14.

8. Mete O, Asa SL. Clinicopathological correlations in pituitary adenomas. Brain Pathology. 2012;22:443-53.

9. Asa SL. Tumors of the Pituitary Gland. Fascicle 15, 4th Series. The Atlas of Tumor Pathology. Washington DC: Armed Forces Institute of Pathology, 2011.

10. Di Ieva A, Davidson JM, Syro LV, Rotondo F, Montoya JF, Horvath E, Cusimano MD, Kovacs K. Crooke's cell tumors of the pituitary. Neurosurgery. 2015;76:616-22.

11. Lindsay JR, Oldfield EH, Stratakis CA, Nieman LK. The postoperative basal cortisol and CRH tests for prediction of longterm remission from Cushing's disease after transsphenoidal surgery. J Clin Endocrinol Metab. 2011;96:2057-64.
12. Witek P, Zieliński G, Szamotulska K, Maksymowicz M, Kamiński G. Clinicopathological predictive factors in the early remission of corticotroph pituitary macroadenomas in a tertiary referral centre. Eur J Endocrinol. 2016;174:539-49.

13. Mete O, Cintosun A, Pressman I, Asa SL. Epidemiology and biomarker profile of pituitary adenohypophysial tumors. Mod Pathol. 2018;31:900-9.

14. Dekkers OM, Horváth-Puhó E, Jørgensen JO, Cannegieter SC, Ehrenstein V, Vandenbroucke JP, Pereira AM, Sørensen HT. Multisystem morbidity and mortality in Cushing's syndrome: A cohort study. J Clin Endocrinol Metab. 2013;98:2277-84.

15. Clayton RN. Mortality in Cushing's disease. Neuroendocrinology. 2010;92 Suppl 1:71-6.

16. Pivonello R, De Leo M, Cozzolino A, Colao A. The treatment of Cushing's disease. Endocr Rev. 2015;36:385-6.

17. Bochicchio D, Losa M, Buchfelder M. Factors influencing the immediate and late outcome of Cushing's disease treated by transsphenoidal surgery: A retrospective study by the European Cushing's Disease Survey Group. J Clin Endocrinol Metab 1995;80:3114-20.

18. Blevins LS Jr, Christy JH, Khajavi M, Tindall GT. Outcomes of therapy for Cushing's disease due to adrenocorticotropinsecreting pituitary macroadenomas. J Clin Endocrinol Metab. 1998;83:63-7.

19. Hague K, Post KD, Morgello S. Absence of peritumoral Crooke's change is associated with recurrence in surgically treated Cushing's disease. Surg Neurol. 2000;53:77-81.

20. Fougner SL, Casar-Borota O, Heck A, Berg JP, Bollerslev J. Adenoma granulation pattern correlates with clinical variables and effect of somatostatin analogue treatment in a large series of patients with acromegaly. Clin Endocrinol (Oxf) 2012;76:96-102.

21. Trouillas J, Roy P, Sturm N, Dantony E, Cortet-Rudelli C Viennet G, Bonneville JF, Assaker R, Auger C, Brue T, Cornelius A, Dufour H, Jouanneau E, François P, Galland F, Mougel F, Chapuis F, Villeneuve L, Maurage CA, Figarella-Branger D, Raverot G; Members of HYPOPRONOS, Barlier A, Bernier M, Bonnet F, Borson-Chazot F, Brassier G, Caulet-Maugendre S, Chabre O, Chanson P, Cottier JF, Delemer B, Delgrange E, Di Tommaso L, Eimer S, Gaillard S, Jan M, Girard JJ, Lapras V, Loiseau H, Passagia JG, Patey M, Penfornis A, Poirier JY, Perrin G, Tabarin A. A new prognostic clinicopathological classification of pituitary adenomas: a multicentric case-control study of 410 patients with 8 years post-operative follow-up. Acta Neuropathol. 2013;126:123-35.

22. Kovacs K, Rotondo F, Horvath E, Syro LV, Di Ieva A, Cusimano MD, Munoz DG. Letter to the editor. Endocr Pathol. 2015;26:93- 